\title{
Information and communication technology in chronic diseases: a patient's opportunity
}

\author{
Simonetta Scalvini $\cdot$ Doriana Baratti $\cdot$ \\ Giuliano Assoni · Margherita Zanardini • \\ Laura Comini · Palmira Bernocchi
}

Received: 1 February 2013/Accepted: 26 June 2013/Published online: 16 July 2013

(C) Springer-Verlag Italia 2013

\begin{abstract}
The increasing aging population, the prevalence of chronic diseases and rising costs have brought about some unique health care challenge to our global society. In response to the unmet health care needs, researchers are actively seeking for innovative solutions that target for (1) prevention of diseases and (2) personalized diagnosis and treatment. It is envisaged that by taking preventive measures for health monitoring, diagnosing and treating patients with a personalized approach at an early stage of disease development, health care will be more cost effective and sustainable. The authors provide an overview of the advancements in Information and Communication Technology (ICT), and explain how some innovative health solutions, through the use of Telemedicine, can now be an opportunity for patients and their family.
\end{abstract}

Keywords Information and Communication Technology · Telemedicine $\cdot$ Ethics

Incidence and prevalence of chronic diseases have increased steadily in recent decades and are continuously growing up. Chronic diseases (such as chronic heart failure,

S. Scalvini $(\varangle)$ - D. Baratti · G. Assoni - M. Zanardini .

P. Bernocchi

Telemedicine Service, Fondazione Salvatore Maugeri,

IRCCS, Institute of Lumezzane, Via Giuseppe Mazzini 129,

25066 Lumezzane, Brescia, Italy

e-mail: simonetta.scalvini@fsm.it

L. Comini

Health Division, Fondazione Salvatore Maugeri,

IRCCS, Institute of Lumezzane, Via Giuseppe Mazzini 129,

25066 Lumezzane, Brescia, Italy
COPD, hypertension, diabetes etc.) are associated with a progressive decrease in quality of life and physical performance; frequent hospitalizations, long period of sick leave, and high rate of illness associated to disability have significant health and macroeconomic consequences [1].

General practice could provide good quality of care for a range of high-prevalence chronic diseases, at the same time providing continuity of care and management of comorbidities. Although quality of care for patients with chronic diseases is improving in general practice, about half of patient care does not meet optimal standards. Many factors contribute to the gap between optimal and current practice and are not related completely to financing system, but are also related to availability of other disciplines to participate in care team, to the limited engagement with self-management education, and lack of information and decision support systems. Patients ask to General Practitioner (GP)'s and specialists continuity of care, emotional support, helping them in the navigation in the health care system and providing self-management support. A deep change is present in the GPs' and specialists' behavior because of increase in the number of patients with chronic diseases, different patient's approach to the disease and a big transformation caused by the use of Information and Communication Technology (ICT) [2].

Aging is a growing issue. Patients with chronic diseases are living longer. ICT and telehealth can help patients to manage their chronic conditions on a day-to-day basis, but it is still too much of a minority. It is the exception rather than the rule. Independent Living and Active Aging are the principle areas of research to expand personalized services for empowering people, with age-related dependencies, to live independently for longer. The target is to develop novel prototypes of systemic solutions compensating for prevailing age-related physical and cognitive impairments 
leading to a significant prolongation of functional capacity, delay in institutionalization, increased autonomy and participation in society.

ICT can improve the quality of life and satisfaction with health care, but it is highly subjective. Patient involvement is crucial and it is still not clear if the benefits are perceived as such by the patients themselves.

Now it is time to provide for an early detection of chronic conditions through intervention based on professional coaching and self-management, employment of ICT tools to enable self-management (e.g., personal health records) and use of a management tool that integrates all aspects of an individual patient's health care, including medical, social and emotional issues.

The sharp increase in the use of ICT tools has encouraged the introduction of innovative health care programs with high technological content, both in the clinical and in the organizational setting [3]. Through efficient ICT networks, adequate medical care can be ensured to chronic patients at home, moreover, the best application and use of available resources can guarantee standardized and efficacious health services [4].

The Chronic Care model was proposed by the World Health Organization [5] almost one decade ago to face the epidemics of chronic conditions. It is widely accepted as a conceptual framework with Integrated Care Services (ICS) being one of the core components, but its implementation represents a profound reshaping of health care. ICT is acknowledged as key enabler of ICS through the development and small-scale validation of personalized services and integrated care programmes, engaging chronic patients and relatives as active members of the care team, enhancing collaboration among careers and promoting seamless continuity of care across different care settings.

\section{Telemedicine}

Telemedicine (TM) is an ICS involving the use of ICT to deliver at distance health care to patients separated from medical provider, can use proper strategies for future extensive regional deployment aiming at transferring complexity from hospital to primary care and to patient home with a proper integration with community services.

The TM approach is of particular importance for elderly or patients which suffer from multiple chronic conditions which can benefit "at distance" from an integrated personalized care approach (i.e., integration between primary, secondary, home and self-care).

The patient and provider delivering care do not have to be in close proximity, and patients can exchange health information or receive health care services in all settings (rural and non-rural). Telemedicine is not a telephone conversation or a fax. Instead, telemedicine typically involves the application of both video and audio technologies in support of health care delivery interacting with a nurse.

Two primary approaches are used in TM: real time and store-and-forward.

In either approaches there is both a patient site and a consult site. The patient site is where the patient is physically located when the video and audio information is gathered (i.e., home or dedicated on-site office). The consult (at distant) site is the physical location of the provider, usually the hospital.

Real time refers to the live presence of both patient and provider in an interactive environment so that the patient is actually seen by the provider and communication between them can take place. Store-and-forward includes delivery of audio or video clips, images or clinical data that are evaluated at a later time (sometimes not more than a few minutes). Store-and-forward applications enable asynchronous communication, with the advantage of not needing concurrent participant involvement.

Two ways of delivery are used in TM: Structured telephone support and Home Telemonitoring.

Structured telephone support is monitoring and patient's self-care management delivered using simple telephone technology under tutoring of a nurse through scheduled phone call appointments; clinical data may have been sent, collected and stored in a computer; support by the nurse can also be provided on demand by patients each time 365 days/year following his/her specific need of assistance.

Home Telemonitoring encompasses the use of audio, video, and other telecommunication technologies to monitor patient status at distance. It is an automated process for the transmission of data on a patient's health status from home to the respective health care setting. Only patients or their family members, when necessary, are responsible for keying in and transmitting their data without the help of a health care provider such as a nurse or a physician.

The ICT systems include several supports and programs:

1. a personal health record, which comprises the personal characteristics of an individual (e.g., clinical profile, preconditions, risk factors, unhealthy behaviors, preferences, physical activity, sleep, mental status, diseases etc.);

2. advanced sensors to acquire data on lifestyle aspects, behavior and surrounding environment, early detection of deterioration, including data acquired by sensors and individual self-assessment (Table 1);

3. wearable, portable, mobile or web-based systems for monitoring of patient status and activity, therapy compliance or treatment at the point of need (Table 1); 
Table 1 Useful definitions in ICT applications

\begin{tabular}{lc}
\hline & Definition \\
\hline Device & An appliance, usually mechanical, designed to perform a \\
& specific function, such as prosthesis or orthesis \\
A & device, usually electronic, which detects a variable quantity \\
& and measures and converts the measurement into a signal to be \\
& recorded elsewhere \\
& They include mobile phones, personal digital assistants, \\
& monitoring devices and wireless devices \\
Mobile systems & They are small handheld tablets designed for mobile Web \\
& browsing and multimedia activities in a lightweight, low-cost \\
Portable technology & device as IPod, IPad, or smarthphone \\
& Sensors for assisting daily living at home (home sensing and \\
& control, bed/chairs sensors \\
Biosensors or device worn on the person's body that \\
communicates with a data acquisition module (Bluetooth/ \\
wireless technology) \\
Data collection, processing and transmission are in connection \\
with an external service for storage and forward repository \\
or patient monitoring device \\
Used primarily for monitoring vital signs as temperature, \\
weight, blood pressure, heart rate, respiratory rate [pulse \\
oximeter], glucose and cholesterol monitor, pedometer \\
Tech togs, or fashion electronics, they are clothing and \\
accessories incorporating computer and advanced electronic \\
technologies (arm band, T-shirts) \\
Web-Based system refers to those applications or services that \\
are resident on a server that is accessible using a Web browser \\
Web-based systems
\end{tabular}

4. auto-adaptive and self-calibrating systems that take into account the acquisition of physiological data in non-clinically controlled environments;

5. decision support systems for professionals and patients, as well as patient guidance services, which build on multimodal data fusion (involving e.g., physiological, environmental, emotional and genetic data), data and pattern analysis, and modeling and predictive algorithms of patient health status (see for example:http://www.Familydoctor.org)

6. stratification of patients to care programs and personalization of such programs to specific characteristics of patients.

Sensors and mobile wireless devices, above mentioned at points 2 and 3, are better described in Table 1. The terms mobile and wireless are often used interchangeably, as mobile implies technologies that are portable and wireless implies that a device can be connected wirelessly to local or wide area networks. The most part of them is already present on the market and could empower people with chronic diseases and age-related dependencies to live independently for longer.

Despite the fact that a review reported that almost 9,000 studies on telecare trials and pilot studies have been published [6], the quality of the evidence-based studies remains limited. The above mentioned review [6], looking at the use of telecare by older people and individuals with long-term conditions, was able to identify 68 randomized controlled trials and a further 30 observational studies with 80 or more participants. The authors concluded that the most effective telecare interventions, including those targeted at individuals with diabetes or heart diseases, appeared to be automated vital signs monitoring (for reducing health service use) and telephone follow-up by nurses (for improving clinical indicators). While the evidence remains mixed, a growing number of studies pointing to the effectiveness of different telehealth interventions in specific contexts and settings can be identified. Potentially, greater investment in telehealth could reduce the use of long-term health services and increase economic benefits covering larger number of patients. Therefore, pilot projects should be designed with evaluation in mind, and consideration given to the integration of telehealth applications within the care system [7]. As we have noted, much less information is available on the economic case for investing in such applications. Quantitative estimates of costs and realizable benefits relying upon extrapolations of outcomes of projects and trials need to be treated with great caution. One key issue, therefore, is to further strengthen the evidence base, both in terms of benefits and effectiveness within routine settings.

The use of ICT can realize systemic solutions compensating age-related physical and cognitive impairments leading to a significant prolongation of functional capacity, delay in institutionalization, increased autonomy and participation in society. We will be able to identify key 
services with high impact on activities of daily living, safety, mobility, social inclusion with seamless support in and outside the home. The further work can help elderly users and their careers, in terms of increasing system efficiency (e.g., easy personalization and adaptation to specific needs and preferences), improving reliability and easy enduser acceptance.

All kinds of actions in health care (i.e., patient registration, medical consultations, medical diagnosis, therapy, drug prescriptions, etc.) are nowadays supported by ICT.

Taken for granted that ICT development had to be in line with basic values of health care from an ethical point of view, the application of these different possibilities in chronic patients has to be considered an opportunity.

Health services are organized around the treatment of acute episodes: inpatients or outpatients are treated due to a specific problem. Nevertheless, patients with chronic pathologies require continuous care to maintain or improve their quality of life. They need rapid access to professional advice when facing psycho-physical changes or new vital circumstances that may arise. Besides, they seek to participate actively in the decisions taken upon their treatment, after gathering information regarding their condition. Nowadays, as a consequence of the fragmentation of health systems, one same patient is seen by various physicians, according to his/her health condition. These professionals do not share information regarding the patient, nor do they have access to a shared case history, and this may lead to the requirement of unnecessary and repeated tests, an increase in unfavorable effect risks, incompatible prescriptions, and, sometimes, contradictory recommendations.

Within this context, the use of ICT will improve the uncoordinated and fragmented manner in which health services are nowadays provided, thus allowing, by means of tools and electronic integrated services, a better health care: more rapid and precise diagnosis, less medical errors and unnecessary tests, optimization of waiting time to receive care and access to test results, and the reduction of administrative expenses.

\section{What about the point of view of the patients and their family on the ICT in the health system?}

From the patients' view, the situation depends on their daily use of ICT, and is totally different from patient to patient; in the last years, we assist to a better acceptance of ICT in health care improving the social capacity of citizens to utilize e-Health technologies for better prevention and treatment in the framework of an aging population [8]. Again, ICT increases patient responsibility for their own health, improve the mobility of citizens with chronic diseases (personal health record on the web), reduced hospital visits and medical consultations; chronic patients are much more involved in their care plan and understand it. The patients' empowerment permits to improve knowledge about the disease and manage some events by themselves, but this involvement are sometimes seen as a great responsibility by the patient and his family [9]. During the last years, a great deal of energy has been devoted to look for evidence of the benefit of ICT in the health scenario. In fact, you can find positive ICT experiences reported by Veterans administration and Kaiser Permanente on a large number of people using an homogeneous ICT network. In contrast, we can also find several well designed controlled studies exploring the role of ICT in different health scenarios with rather negative results. However, the increasing penetration of ICT in health care is out of any reasonable dispute and it will only keep growing in the forthcoming years. The trend is strongly linked to the urgent needs to: increase accountability of health care organizations, make use of huge amount of health information to generate knowledge and, ultimately, improve care, and, reshape the health system overcoming the current fragmentation. We currently have enough information to know that ICT alone is not useful to generate benefits, but the use of ICT is a must in the process of redesign novel health care services that will be generating efficiencies and doing so, the ICT will foster the needed change in health care. In other words, the current dilemma is not ICT yes or not, but how to use it in a mature and balanced manner such that it makes possible to enhance the health outcomes for chronic patients.

\section{Conflict of interest None.}

\section{References}

1. Scalvini S, Giordano A (2012) Heart failure: optimal postdischarge management of chronic HF. Nat Rev Cardiol 10(1):9-10

2. Harris Mark F, Zwar Nicholas A (2007) Care of patients with chronic disease: the challenge for general practice. MJA 187: 104-107

3. While A, Dewsbury G (2011) Nursing and information and communication technology (ICT): a discussion of trends and future directions. Internal J Nur Stud 48(10):1302-1310

4. Wootton R (2012) Twenty years of telemedicine in chronic disease management-an evidence synthesis. J Telemed Telecare 18(4): 211-220

5. Coleman K, Austin BT, Brach C, Wagner EH (2009) Evidence on the chronic care model in the new millennium. Health Aff 28(1): $75-85$

6. Barlow J, Singh D, Bayer S et al (2007) A systematic review of the benefits of home telecare for frail elderly people and those with long-term condition. J Telemed Telecare 13(4):172-179

7. Barlow J, Bayer S, Curry R (2003) The design of pilot telecare projects and their integration into mainstream service delivery. J Telemed Telecare 9(Suppl 1):S1-S3

8. Collste G (2008) Applied Ethics and ICT-Systems in Healthcare. In: Duqnoi P, George C, Kimppa K (eds) Ethical, legal, and social issues in medical informatics. Medical Information Science 
Reference. Hershey, New York, pp 29-47. (www.igi-global.com/ reference/details.asp?id=7741)

9. Collste G, Duquenoy P, George C, Hedström K, Kimppa K, Mordini (2006) ICT in medicine and health care: assessing social, ethical and legal issues. Social informatics: an information society for all? In: processing of remembrance of rob kling IFIP international federation for information 223:297-308 